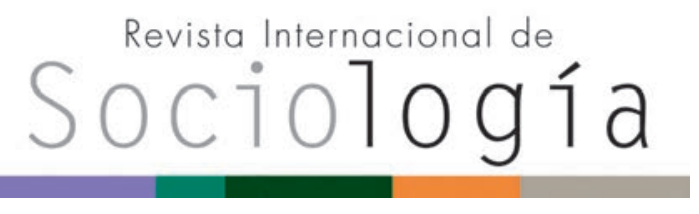

Revista Internacional de Sociología RIS

vol. 76 (3), e105, julio-septiembre, 2018, ISSN-L:0034-9712

https://doi.org/10.3989/ris.2018.76.3.16.161

\section{DIFERENCIAS DE GÉNERO EN LA DISTRIBUCIÓN DEL TIEMPO DE TRABAJO EN LAS REGIONES ESPAÑOLAS}

\author{
Amaia AltuZARRA Artola \\ University of the Basque Country (UPV/EHU) \\ amaia.altuzarra@ehu.eus \\ ORCID iD: https://orcid.org/0000-0002-9256-0050
}

\section{CatAlina GÁlvez GÁlVEZ}

University of the Basque Country (UPV/EHU)

catalina.galvez@ehu.eus

ORCID iD: https://orcid.org/0000-0001-7398-8599

\author{
Ana María GonzÁlez FloRes \\ University of the Basque Country (UPV/EHU) \\ ana.gonzalezflores@ehu.eus \\ ORCID iD: https://orcid.org/0000-0002-8228-5868
}

Cómo citar este artículo / Citation: Altuzarra Artola, A., C. Gálvez Gálvez y A. M. González Flores. 2018. "Diferencias de género en la distribución del tiempo de trabajo en las regiones españolas". Revista Internacional de Sociología 76(3): e105. https://doi.org/10.3989/ris.2018.76.3.16.161

\section{RESUMEN}

Este trabajo examina la relación entre los patrones de distribución del tiempo dedicado al trabajo remunerado y al doméstico de las parejas heterosexuales y las características socioeconómicas e institucionales de las regiones españolas. La hipótesis que contrastamos es que las regiones con un sistema institucional amplio de políticas sociales y familiares, mayor riqueza, nivel educativo y participación femenina en el mercado laboral, tienen una distribución del trabajo remunerado y doméstico entre géneros más igualitaria. Distinguimos, dentro del trabajo doméstico, entre el tiempo dedicado a las tareas del hogar y al cuidado de los niños. Los datos utilizados provienen de la última Encuesta de Empleo de Tiempo española (2009-2010). La metodología utilizada es el Análisis en Componentes Principales (ACP) y el Análisis Clúster. Los resultados permiten identificar cinco grupos de regiones o patrones de distribución del tiempo entre hombres y mujeres que se asocian con contextos socioeconómicos e institucionales diferentes.
\end{abstract}

\section{Palabras Clave}

Cuidado de hijos; Marco institucional; Regiones; Trabajo doméstico.

\section{GENDER DIFFERENCES IN THE ALLOCATION OF WORKING TIME IN THE SPANISH REGIONS}

Copyright: (C) 2018 CSIC. Este es un artículo de acceso abierto distribuido bajo los términos de la licencia de uso y distribución Creative Commons Reconocimiento 4.0 Internacional (CC BY 4.0).

Recibido: 09/11/2016. Aceptado: 07/11/2017

\section{Abstract}

This study examines, from a regional perspective, the relationship between the patterns of time allocation to paid and domestic work within heterosexual couples and the socio-economic and institutional characteristics of regions. The hypothesis of this work is that, in regions with an institutional system characterized by generous social and family policies, greater participation of women in the labor market, greater wealth and higher educational level, the distribution of paid and domestic work between men and women is more egalitarian. Regarding domestic work, we distinguish between time devoted to housework and childcare. Using the 20092010 Spanish Time Use Survey, we applied Principal Components Analysis (PCA) and Cluster Analysis. We identified five groups of regions or patterns of time allocation between men and women. Heterogeneity among these patterns can be associated to regional differences in the economic, social and institutional settings.

\section{KEYWORDS}

Childcare; Domestic work; Institutional framework; Regions. 


\section{INTRODUCCIÓN}

Uno de los ámbitos donde se ponen de manifiesto importantes desigualdades de género es en la distribución del uso del tiempo. Los hombres dedican más tiempo que las mujeres al trabajo fuera del hogar (trabajo remunerado), en tanto que las mujeres continúan siendo las principales responsables del trabajo doméstico, entendiendo este trabajo como la realización de tareas del hogar y el cuidado de niños, ancianos, personas discapacitadas o cuidados personales de adultos. Esta especialización también se produce dentro de las distintas tareas que comprenden el trabajo doméstico. Las mujeres, habitualmente, se encargan de las tareas asociadas a horarios poco flexibles y más rutinarias, tanto en el hogar (limpieza del hogar, cocinar, planchar, etc.) como en el cuidado de los niños (preparación de comidas, baño, etc.) (Gracia y Esping-Andersen 2015; Esping-Andersen et al. 2013; Hook 2010).

Aunque la desigualdad de género respecto al uso del tiempo está prácticamente extendida en todos los países occidentales, la amplitud de la brecha de género varía de unos países a otros en función del régimen de bienestar social, las políticas de empleo y familiares, las tasas y beneficios del sistema y las normas sociales (Anxo et al. 2011). Son muchos los estudios que destacan la influencia del contexto socioeconómico e institucional sobre el tiempo que mujeres y hombres dedican al trabajo remunerado y doméstico (Gracia y Esping-Andersen 2015; EspingAndersen et al. 2013; Hook 2010, 2006; Lewis 2009; Davis y Greenstein 2004; Fuwa 2004).

En España, los patrones de distribución del tiempo entre hombres y mujeres siguen, "groso modo", las tendencias apuntadas. No obstante, la brecha de género puede estar ocultando realidades regionales ${ }^{1}$ muy diversas que tienen su origen en los diferentes contextos económicos, sociales, institucionales y culturales, así como en las medidas de política social, familiar y de género implementadas por los gobiernos regionales. España se caracteriza por poseer un sistema político altamente descentralizado en el que los gobiernos regionales tienen amplios poderes para definir sus objetivos de política social, familiar y de igualdad. Esta competencia ha favorecido que las regiones desarrollen y consoliden instituciones y políticas orientadas a reducir las diferencias de género dentro de su territorio. Pese a que existen algunas similitudes en las estrategias de género de las regiones, debidas a la influencia de las orientaciones de la Unión Europea y del propio gobierno central, en España coexisten una gran variedad de políticas de género (Bustelo 2014; Bustelo y Ortbals 2007).

El objetivo de este trabajo es examinar, desde una perspectiva regional, la relación entre los patrones de distribución del tiempo dedicado al trabajo remunerado y doméstico dentro de las parejas heterosexuales y las características socioeconómicas e institucionales de las regiones. Para ello, utilizamos datos de la última oleada de la Encuesta de Empleo del Tiempo para el periodo 2009-2010 y tomamos como referencia exclusivamente aquellas personas con edades comprendidas entre los 25 y 65 años que viven en pareja. De este análisis, esperamos que las regiones donde las parejas distribuyen de manera más equitativa el trabajo remunerado y doméstico, sean aquellas con mayor desarrollo económico, políticas sociales y familiares más generosas y una más elevada participación de la mujer en el mercado laboral.

Este trabajo contribuye a la literatura empírica sobre el uso del tiempo de diferentes formas. En primer lugar, proporciona evidencia empírica sobre la influencia del contexto económico-social e institucional en la distribución del tiempo entre géneros desde una perspectiva regional. Esta cuestión ha sido estudiada comparando grupos de países (Bettio et al. 2013; Lahey y De Villota 2013; Esping-Andersen et al. 2013; Giménez-Nadal y Sevilla 2012; Anxo et al. 2011; Gálvez-Muñoz et al. 2011; Hook 2010), pero se ha abordado escasamente el estudio de las diferencias regionales dentro de un mismo país. Cabe señalar los trabajos de Geist (2009) para Alemania, y de Ruppanner y Maume (2016) para Estados Unidos. El estudio de las diferencias de género por países es procedente cuando se trata de países internamente homogéneos y con marcos institucionales centralizados. Sin embargo, los países grandes tienden a ser más heterogéneos en términos culturales, laborales, sociales y económicos. Las diferencias regionales, además, se acentúan cuando el poder del Estado está descentralizado. En segundo lugar, se aplica el Análisis en Componentes Principales (ACP), una técnica estadística poco utilizada en los análisis sobre el uso del tiempo, que permite visualizar la información de una manera más gráfica e intuitiva. Finalmente, la información disponible en la Encuesta de Empleo del Tiempo se cruza con una amplia variedad de datos obtenidos en otras fuentes estadísticas, relativos a las distintas regiones (datos del mercado laboral, estructura productiva, riqueza económica y políticas sociales), que permiten obtener una visión más completa.

El resto del trabajo se organiza de la siguiente manera: en la sección 2 se realiza una revisión de los estudios empíricos que analizan el uso del tiempo desde la perspectiva institucionalista; en la sección 3 se presentan los datos que se van a explotar y se explica la metodología seguida; la sección 4 se dedica a explicar los principales resultados obtenidos, y finalmente, se presentan las conclusiones fundamentales.

\section{REVISIÓN DE LA LITERATURA}

La desigual distribución del uso del tiempo entre hombres y mujeres ha sido estudiada desde diferentes enfoques teóricos. Un primer enfoque es el de la 
disponibilidad de tiempo, que sugiere que el tiempo dedicado al trabajo doméstico se realiza racionalmente de acuerdo con la disponibilidad que tienen los miembros del hogar para acometer las tareas (Coverman 1985). Una variante de este enfoque es la teoría de la especialización (en el hogar) de Becker (1981), que considera las ventajas comparativas entre hombres y mujeres en la determinación de la división del trabajo entre ambos. Un segundo enfoque es el de los recursos relativos (o del poder de negociación) según el cual la distribución del trabajo doméstico es un reflejo de las relaciones de poder entre hombres y mujeres (McElroy y Horney 1981; Manser y Brown 1980; Lundber y Pollack 1996). El tercer enfoque, basado en la ideología de género, presenta una alternativa a los planteamientos anteriores. Este paradigma considera que las normas sociales atribuyen unos roles a las mujeres y hombres en la división de las tareas del hogar. De la mujer se espera un rol de dedicación al hogar y al cuidado de niños y personas dependientes; dedicación que aumenta entre las mujeres que viven en pareja y con hijos (Bittman et al. 2003; Greenstein 1996; Brines 1994; West y Zimmerman 1987).

Frente a estos planteamientos teóricos que se basan en las características individuales de los hombres y mujeres para explicar la desigual distribución del tiempo entre los miembros del hogar, ha surgido un cuarto enfoque denominado institucionalista, el cual sostiene que la desigualdad de género es muy sensible al entorno socioeconómico e institucional de los países en que residen las familias. El estado de bienestar, las políticas de apoyo a la familia (conciliación), la participación de la mujer en el mercado laboral, el desarrollo económico y los valores y el contexto cultural de cada país influyen en el tiempo que mujeres y hombres dedican al trabajo doméstico (Gracia y Esping-Andersen 2015; Esping-Andersen et al. 2013; Hook 2010, 2006; Lewis 2009; Davis y Greenstein 2004; Fuwa 2004).

Siguiendo este enfoque teórico, varios trabajos se han ocupado de estudiar la relación entre el uso del tiempo y las características de los sistemas de bienestar social. Estos estudios se inspiran en el trabajo seminal de Esping-Andersen (1990), en el que se analizan los modos en los que se reparte la producción de bienestar entre los hogares, el mercado y el Estado. Este autor divide un conjunto de países en tres grupos según su régimen de bienestar. En primer lugar, estarían los países socialdemócratas, en los que el Estado protagoniza un papel importante, con una fuerte integración de las políticas sociales y económicas. En este grupo se encontrarían Suecia y Noruega. En un segundo grupo estarían los regímenes liberales, en los que la intervención estatal está claramente subordinada al mercado y, por tanto, los individuos deben acudir al mismo para satisfacer sus necesidades. Es el caso de países como Estados
Unidos, Reino Unido, Canadá y Australia. Por último, un tercer grupo de países estaría caracterizado por regímenes que vinculan el acceso al bienestar a la clase y al estatus social. En este grupo de países más conservadores, la familia tradicional tiene una gran importancia y el Estado dota de recursos sociales sólo cuando la capacidad de la familia para hacerlo se agota. En este bloque se encontrarían países como Alemania, Francia e Italia. Con posterioridad, este modelo fue criticado desde el ámbito feminista por no incluir las relaciones de género en los resultados de las políticas desarrolladas ni considerar el papel del Estado en las relaciones de género, desigualdad y poder (Sainsbury 1996; Orloff 1996).

Gracia y Esping-Andersen (2015) y EspingAndersen et al. (2013), utilizando datos de Dinamarca, Reino Unido y España, muestran que el tiempo que los hombres dedican a las tareas del hogar y al cuidado de los niños difiere entre países con distintas normas de género, políticas familiares y tasa de empleo de las madres. Como consecuencia, Dinamarca, que está a la vanguardia en el empleo femenino, dispone de políticas familiares universales y condiciones que favorecen la conciliación familiar, es la sociedad más igualitaria. Hook $(2010,2006)$ compara en su trabajo 19 países, constatando que, en países donde hay más servicios públicos destinados al cuidado de los niños, los hombres dedican más tiempo al hogar y al cuidado de los niños, los padres tienen la posibilidad de optar a una excedencia de paternidad y también es más elevada la tasa de participación de la mujer en el mercado laboral. Asimismo, otros estudios sugieren que la participación de los hombres en el trabajo del hogar es mayor en aquellos países en los que la mujer posee más poder político y económico (Fuwa 2004) y en Estados políticamente más liberales y en familias menos tradicionales (Ruppanner y Maume 2016).

En el mismo sentido, Gálvez-Muñoz et al. (2011), utilizando datos de la Encuesta Europea Armonizada de Empleo del Tiempo y con técnicas Clúster, identifican cuatro grupos de países europeos. Los países con una distribución de género más igualitaria son aquellos donde el mercado de trabajo funciona mejor, remunerando una parte mayor del trabajo de acuerdo con la productividad marginal y el capital humano, como en Reino Unido, o donde existe un sistema de bienestar avanzado que permite desplazar parte del trabajo doméstico y cuidado de los niños al Estado, como ocurre en los países escandinavos. Los países mediterráneos (Italia, España y Portugal) son los que tienen un reparto del tiempo de trabajo más desigual. Estos países registran la tasa de actividad femenina más baja y la familia posee la máxima responsabilidad en la provisión del bienestar. Los países del este de Europa constituyen, por su parte, un grupo muy heterogéneo.

En la misma línea, Anxo et al. (2011) analizan las diferencias de género en el uso del tiempo a lo lar- 
go del ciclo de vida en varios países (Francia, Italia, Suecia y Estados Unidos). Estos autores, basándose en los datos de las respectivas encuestas nacionales del uso del tiempo realizadas a finales de los años noventa y principios de la década del 2000, y a través de un estudio de sección cruzada, constatan que los países que presentan una menor desigualdad de género son aquellos que desarrollan, entre otros factores, políticas activas que promueven la igualdad, como, por ejemplo, un sistema flexible de baja maternal o la provisión de servicios públicos destinados al cuidado de los niños.

Burda, Hamermesh y Weil (2013), por su parte, estudian la relación entre la riqueza del país y la brecha de género en relación con el tiempo dedicado al trabajo total en 27 países, y encuentran que la relación es negativa. Además, sugieren que el tiempo dedicado al trabajo por mujeres y hombres en países ricos no católicos es similar (iso-work); sin embargo, en los países católicos, al igual que en los países más pobres, las mujeres dedican más tiempo al trabajo que los hombres. El desarrollo de normas sociales constituye una de las causas explicativas de las diferencias de género. Similares conclusiones obtienen Giménez-Nadal y Sevilla (2012) al examinar las diferencias de género en siete países desarrollados (Australia, Canadá, Finlandia, Francia, Países Bajos, Noruega y Reino Unido).

Los trabajos empíricos para el caso español han arrojado conclusiones consistentes con los resultados anteriores en relación a la importancia del contexto social e institucional en la brecha de género y su evolución. Usando datos de las dos oleadas disponibles de la "Encuesta de Empleo de Tiempo española” (2002-2003 y 2009-2010), Giménez-Nadal y Sevilla (2014) analizan los cambios registrados en la distribución del tiempo en la población española, no encontrando evidencia de iso-work. Detectan que las mujeres, en total, trabajan más horas que los hombres y, en particular, en el trabajo doméstico. España, dentro del grupo de países mediterráneos, se caracteriza por la presencia de rígidas normas sociales (Giménez-Nadal y Sevilla 2012), con una baja presencia del sector público en los servicios relacionados con el cuidado de los niños menores de tres años (Gutiérrez-Domenech 2005) y que fomenta el modelo male-breadwinner (Lewis 2009; EspingAndersen 1999).

Por otro lado, la incorporación de la mujer al mercado laboral ha alterado la distribución del tiempo dedicado al trabajo remunerado y doméstico entre hombres y mujeres. Algunos autores, como Ajenjo y García (2014), utilizando los datos de las dos encuestas sobre el empleo del tiempo de España, constatan que a lo largo de la primera década del siglo XXI las parejas españolas han reducido la brecha de género en cuanto al uso del tiempo, sobre todo en el trabajo remunerado y en las tareas domésticas ru- tinarias. Este reparto del tiempo más igualitario se ha visto favorecido por el fuerte incremento del número de parejas en las que sólo trabaja la mujer y también de las parejas de doble ingreso.

Otros autores, como Carrasco y Domínguez (2011), sugieren que las nuevas condiciones sociales en España (tales como el aumento de la tasa de empleo femenina y los cambios en la estructura de las familias) han contribuido notablemente a que la mujer disminuya el tiempo dedicado a las tareas del hogar. Sin embargo, sólo han favorecido ligeramente el aumento de la participación del hombre en las tareas del hogar. Por consiguiente, el papel de la mujer como proveedora de los cuidados familiares continúa predominando, de modo que, cuando una mujer reduce el tiempo dedicado al trabajo doméstico no es porque el hombre se haya responsabilizado de ese trabajo, sino porque parte de ese trabajo se ha externalizado, reemplazándolo con el trabajo de otras personas (Gálvez-Muñoz et al. 2011). Este resultado es consistente con la evidencia empírica a nivel internacional: Aguiar y Hurst (2007) examinan el caso de Estados Unidos, y Giménez-Nadal y Sevilla (2012) lo acontecido en siete países industrializados (Australia, Canadá, Finlandia, Francia, Países Bajos, Noruega y Reino Unido). Sus resultados indican que las mujeres han reducido el tiempo destinado a las tareas domésticas que, sin embargo, no ha sido compensado por un aumento similar por parte de los hombres.

Los estudios referidos ponen de manifiesto el papel que juegan los factores institucionales a la hora de explicar la brecha de género en el uso del tiempo en un país. En países grandes como España, las regiones que lo componen comparten algunas características institucionales y culturales. No obstante, también tienden a ser más heterogéneos, especialmente en términos económicos y sociales. Estas diferencias regionales, además, se amplían cuando el poder del Estado está descentralizado, como es el caso que nos ocupa, en el que los gobiernos regionales poseen amplias competencias en materia económica, social y de igualdad. Es por ello que parece razonable realizar un análisis a nivel regional, para entender cómo factores institucionales y socio-económicos pueden estar asociados con una amplitud determinada de la brecha de género en el uso del tiempo.

Este trabajo pretende, por tanto, contrastar la hipótesis de que las regiones españolas que poseen un sistema institucional más amplio de políticas sociales y familiares, mayor presencia de la mujer en el mercado laboral, mayor riqueza económica y mayor nivel educativo, tienen una distribución más igualitaria en el reparto de trabajo remunerado y doméstico (tareas del hogar y cuidado de los hijos) en las parejas heterosexuales. 


\section{DATOS Y METODOLOGÍA}

\section{Datos}

En este trabajo utilizamos datos de la última Encuesta de Empleo del Tiempo (EET) (2009-2010) para España, realizada por el Instituto Nacional de Estadística (INE). La población objeto de la encuesta son los hogares que residen en viviendas familiares principales y el conjunto de personas de 10 o más años que viven en el hogar. El total de hogares encuestados es de 11.538 .

La encuesta emplea el método del diario para recoger datos del uso del tiempo. Los miembros del hogar de 10 y más años deben registrar en un diario la actividad que realizan en cada momento. El diario se divide en intervalos de diez minutos y los entrevistados tienen que registrar la actividad principal que están haciendo en ese momento y cualquier otra actividad que estén realizando simultáneamente, la cual es denominada actividad secundaria.

La actividad principal se codifica mediante una clasificación que sigue un orden jerárquico de diez actividades que incluyen: (1) cuidados personales (necesidades fisiológicas, cuidado personal, dormir), (2) trabajo remunerado o comprometido, (3) estudios, (4) trabajo no remunerado (hogar y familia), (5) trabajo voluntario y reuniones, (6) vida social y diversión, (7) deportes y actividades al aire libre, (8) aficiones e informática, (9) medios de comunicación y (10) desplazamientos (trayectos y tiempo no especificado). Conviene anotar que tomamos la media del tiempo dedicado a cada actividad durante los días laborables, los fines de semana y las vacaciones, creando así un día medio.

Además de esta información, la EET ofrece datos sobre ingresos anuales, nivel de educación y número de hijos, entre otras variables. De toda la información contenida en la EET utilizamos una sub-muestra de 1.087 hogares $^{2}$, compuesta por:

- Parejas heterosexuales, casadas o conviviendo de hecho, con hijos menores de 10 años o sin hijos. Los hogares con adultos diferentes a la pareja se han excluido para evitar un potencial efecto de la ayuda de otros adultos en el cuidado de los niños o en las labores del hogar.

- $\quad$ Personas con edades comprendidas entre 25 y 65 años. De esta manera evitamos incluir al grueso de estudiantes y jubilados.

Finalmente, para contextualizar y completar mejor los rasgos de las regiones, se ha recurrido a información sobre el mercado laboral, la estructura económica, la renta y las políticas de conciliación y gasto social de las regiones ${ }^{3}$. Esta información se ha obtenido del Instituto Nacional de Estadística (INE), del Ministerio de Empleo y Seguridad Social, de la Fundación BBVA-IVIE (Instituto Valenciano de Investigaciones Económicas) y del Instituto de Política Familiar.

\section{Metodología}

La metodología utilizada es el Análisis de Componentes Principales (ACP) ${ }^{4}$. Esta técnica, ampliamente utilizada en diversas disciplinas, es una herramienta muy útil para estudiar los diferentes patrones regionales en relación con el uso del tiempo entre hombres y mujeres.

El ACP es un método multivariante que permite transformar un conjunto de datos multidimensional en otro de reducidas dimensiones. Básicamente, el ACP descompone el universo de datos y crea un número de variables independientes, denominadas componentes principales o factores, que son una combinación lineal de las variables originales. La elección de los factores se realiza de tal forma que el primero recoja la mayor variabilidad de los datos y explique mejor el comportamiento general de los mismos, permitiendo interpretar las similitudes entre los individuos con pérdida de información mínima. Cada componente principal posterior recoge la variabilidad remanente de dichos datos.

En el ACP, los datos pueden proyectarse sobre un plano factorial de reducidas dimensiones, de manera que se pueden identificar las características asociadas a cada uno de los ejes factoriales con la máxima varianza. El software utilizado es el SPAD 7.0.

El ACP se completa con un Análisis Clúster, que agrupa a los individuos en grupos o clases y sirve para identificar los patrones de uso del tiempo entre hombres y mujeres en las regiones españolas.

\section{Variables activas}

Para los propósitos de este trabajo hemos seleccionado siete variables activas (i.e., variables que participan en la formación de los factores) que pretenden capturar diferencias en la división del tiempo dentro de las parejas, teniendo en cuenta algunas características de los hogares y de los individuos (Tabla I). Las relaciones entre las variables consideradas han sido ampliamente utilizadas en la literatura (Phipps et al. 2011; Killewad y Gough 2010; Craig y Bittman 2008; Craig 2006).

Los ingresos mensuales de los hogares están codificados en la EET en cuatro tramos: menos de 1.200 $€$, entre $1.201 €$ y $2.000 €$, entre $2.001 €$ y $2.500 €$ y más de $3.000 €$. El nivel de estudios se ha codificado en cuatro modalidades: estudios de ESO o inferiores, FP básica o Bachiller, FP superior y estudios universitarios y superiores. Finalmente, el número de hijos presenta otras cuatro modalidades: sin hijos, con un hijo, con dos hijos y con tres o más hijos. Las modalidades de estas tres variables toman los valores 1 , 
Tabla I.

Variables activas para todas las regiones

\begin{tabular}{|lcc|}
\hline & Media & D.S \\
\hline Ingresos del hogar & 1,83 & 0,41 \\
Nivel de estudios (mujer) & 2,38 & 0,22 \\
Número de hijos & 1,51 & 0,13 \\
Nivel de estudios (hombre) & 2,23 & 0,20 \\
Ratio (mujer/hombre) del tiempo dedicado a los hijos & 1,92 \\
Ratio (mujer/hombre) del tiempo dedicado al hogar & 2,32 & 0,32 \\
Ratio (mujer/hombre) del tiempo dedicado al trabajo remunerado & 0,53 & 0,43 \\
\hline
\end{tabular}

Fuente: Elaboración propia

2, 3, y 4, respectivamente. Dado que las categorías de estas variables siguen una secuencia creciente, se han considerado como variables continuas para poder ejecutar el ACP.

\section{Variables ilustrativas}

Adicionalmente, hemos incluido en el análisis un conjunto de variables ilustrativas para caracterizar con mayor detalle las desigualdades de género. Estas variables no participan en la formación de los factores, pero ayudan a la caracterización de las regiones. Las variables ilustrativas consideradas hacen referencia a los gastos sociales, a las políticas familiares y a las características estructurales y laborales de las regiones. La Tabla A.I del Apéndice recoge las variables ilustrativas incluidas.

Dada la dificultad de obtener información cuantitativa comparable sobre los recursos destinados a los hogares por parte de los gobiernos regionales, hemos optado por incluir información cualitativa que resuma la estructura de apoyo a las familias que tienen previstas las distintas CC. AA. (Tabla II). Conviene apuntar que en España coexisten tres niveles administrativos: la Administración General del Estado, las CC. AA. y las Corporaciones Locales, y que esta división competencial se traduce en que las ayudas que reciben las familias no sean las mismas en todas las regiones.

Las ayudas regionales a las familias pueden adoptar dos formas: ayudas directas (pago que abona la Administración a la unidad familiar) y deducciones fiscales ${ }^{5}$. En relación con las primeras, la mayoría de las CC. AA. dispone de ayudas dirigidas a familias con rentas bajas, esto es, se trata de un apoyo más asistencial. Solo siete regiones (Andalucía, Cantabria, Cataluña, Galicia Navarra, País Vasco y La Rioja) ofrecen ayudas que llegan a casi todas las familias.

La conciliación entre la vida laboral y familiar tampoco tiene la misma consideración en todas las regiones. Una proporción significativa de CC. AA. no contempla apoyos para este fin. Andalucía dispone de ayudas universales, mientras que Canarias,
Castilla y León, Cataluña, Galicia, Murcia y Valencia proporcionan ayudas con límite de renta.

Respecto al tratamiento fiscal de los hijos, sólo Cataluña, Galicia, Navarra y País Vasco ofrecen deducciones fiscales universales para los hijos recién nacidos o adoptados. Aragón, Baleares y La Rioja proporcionan ayudas sólo a algunas familias (con el segundo o tercer hijo, etc.) y en Canarias, Castilla-La Mancha, Castilla y León, Madrid y Valencia se otorgan deducciones con límite de renta. En el resto no se contemplan deducciones por hijos.

\section{Resultados}

\section{Análisis descriptivo univariante}

La Tabla III muestra la ratio mujer/hombre en la distribución del tiempo entre trabajo remunerado y doméstico (tareas del hogar y cuidado de los hijos) en las regiones españolas para la submuestra objeto de estudio. Esta ratio proporciona una medida de la desigualdad de género en cada una de las tareas. Si la ratio es inferior a 1 , indica que los hombres dedican más tiempo que las mujeres a la actividad correspondiente. Si es superior a 1 , son las mujeres las que dedican más tiempo a esa actividad. Y sólo cuando alcanza el valor de 1 existe equidad en la distribución de tiempo entre géneros.

Se puede apreciar, en primer lugar, que en todas las regiones las mujeres dedican al trabajo total (remunerado y doméstico) un tiempo mayor que los hombres. En segundo lugar, en todas las regiones los hombres dedican más tiempo al trabajo remunerado que al doméstico, mientras que en el caso de las mujeres ocurre lo contrario. Las mujeres en España trabajan fuera del hogar la mitad de horas que los hombres (la ratio es 0,53 ). Las regiones con ratios más próximas a 1, esto es, con menos desigualdad de género en cuanto al tiempo dedicado al trabajo remunerado son Navarra $(0,72)$, Cataluña $(0,68)$ y La Rioja $(0,66)$. En situación contraria se encuentran Murcia $(0,29)$, Castilla y León y Asturias $(0,35)$. Sin embargo, las diferencias más significativas entre hombres y mujeres 
Tabla II.

Ayudas y deducciones fiscales

\begin{tabular}{|lcccc|}
\hline & Ayudas Familiares (a) & Conciliación (b) & Ayudas guardería (c) & Deducción por hijos (d) \\
\hline Andalucía & 3 & 3 & 1 & 1 \\
Aragón & 2 & 1 & 0 & 3 \\
Asturias & 2 & 1 & 0 & 1 \\
Baleares & 2 & 1 & 1 & 3 \\
Canarias & 2 & 2 & 1 & 2 \\
Cantabria & 3 & 1 & 1 & 1 \\
Castilla y León & 1 & 2 & 1 & 2 \\
Castilla-La Mancha & 1 & 0 & 2 \\
Cataluña & 3 & 1 & 1 & 4 \\
C Valenciana & 1 & 2 & 1 & 2 \\
Extremadura & 1 & 2 & 1 & 1 \\
Galicia & 3 & 1 & 1 & 4 \\
Madrid & 2 & 2 & 1 & 2 \\
Murcia & 1 & 1 & 1 & 1 \\
Navarra & 3 & 2 & 1 & 4 \\
País Vasco & 3 & 2 & 0 & 4 \\
La Rioja & 3 & 2 & 0 & 3 \\
Ceuta y Melilla & 2 & 1 & 1 \\
\hline
\end{tabular}

Notas: (a) 1: todas las ayudas son para familias de renta baja, 2: la mayoría de las ayudas son para familias de renta baja; 3 : las ayudas alcanzan a casi todas las familias.

(b) 1: no existen ayudas; 2: ayudas condicionadas a la renta; 3 : ayudas universales.

(c) 0: no existen ayudas; 1 : sí existen ayudas.

(d) 1: no existen deducciones; 2: deducciones condicionadas a la renta; 3: las deducciones alcanzan a algunas familias; 4: deducciones universales.

Fuente: Instituto de Política Familiar (2015). Elaboración propia

se encuentran en el tiempo que dedican al trabajo doméstico, en especial a las tareas del hogar. La ratio del trabajo doméstico es superior a 1 en todas las regiones y, como media, las mujeres dedican a esta actividad más del doble de tiempo que los hombres. Las regiones con mayores ratios, esto es, con mayor desigualdad de género son Murcia $(2,84)$, Canarias $(2,64)$ y Ceuta y Melilla $(2,55)$. En cambio, País Vasco $(1,63)$, La Rioja $(1,69)$ y Navarra $(1,85)$ muestran la menor desigualdad. Esto evidencia que los hombres continúan especializándose en actividades propias de la economía de mercado, mientras que las mujeres tienden a especializarse en las relativas a la economía de no mercado (Gálvez et al. 2011).

Por lo que se refiere al tiempo dedicado a las tareas del hogar, la mayor brecha de género se registra en Murcia donde las mujeres dedican más del triple de tiempo que sus parejas a esta actividad $(3,43)$. Le siguen Canarias $(2,83)$ y Asturias y Ceuta y Melilla $(2,75)$. País Vasco $(1,57)$, La Rioja $(1,84)$ y Navarra $(1,97)$ son las regiones con una menor desigualdad. En el cuidado de los niños, la brecha de género tiende a ser inferior. De nuevo, Murcia $(2,47)$ muestra la mayor desigualdad de género, junto a Castilla y León $(2,41)$. En el polo opuesto, se encuentran La Rioja $(1,50)$, Cataluña $(1,55)$ y País Vasco $(1,60)$.

\section{Resultados e interpretación del ACP}

Para comprobar la conveniencia de aplicar el ACP se ha evaluado la significatividad del modelo factorial. El análisis realizado constata que las variables están altamente correlacionadas, lo que es indicativo de que existe información redundante y que, al menos, hay un factor común que motiva la aplicación de esta técnica. (Véase Apéndice II).

En la aplicación del ACP se han retenido todos los componentes principales (factores) con un valor propio (varianza) mayor que la unidad. Como consecuencia, tal y como indica la Tabla IV, se han conservado los primeros dos componentes principales, que explican el $70,3 \%$ de la varianza total de los datos originales, proporción que se considera adecuada.

Para interpretar los resultados del ACP resulta clave analizar las correlaciones entre las variables originales y los componentes principales retenidos. La tabla $\mathrm{V}$ presenta las correlaciones (cargas) entre las variables activas y los primeros dos componentes. Las cargas factoriales expresan el peso que cada variable asigna a cada factor. Cargas altas indican que dicha variable es representativa para dicho factor. Como se puede observar, todas las variables tienen correlaciones que exceden 0,40 en valor absoluto, lo que se considera suficientemente significativo para el análisis (Stevens 1986). 
Tabla III.

Brechas de género en el uso del tiempo

\begin{tabular}{|c|c|c|c|c|c|c|}
\hline & $\begin{array}{l}\text { Número de hogares } \\
\text { en la sub-muestra }\end{array}$ & $\begin{array}{c}\text { Trabajo } \\
\text { Total }\end{array}$ & $\begin{array}{c}\text { Trabajo } \\
\text { remunerado }\end{array}$ & $\begin{array}{c}\text { Trabajo } \\
\text { doméstico }\end{array}$ & $\begin{array}{l}\text { Tareas } \\
\text { hogar }\end{array}$ & $\begin{array}{l}\text { Cuidado } \\
\text { hijos }\end{array}$ \\
\hline Andalucía & 120 & 1,34 & 0,55 & 2,12 & 2,41 & 1,84 \\
\hline Aragón & 45 & 1,25 & 0,61 & 2,05 & 2,16 & 1,91 \\
\hline Asturias & 37 & 1,25 & 0,35 & 2,24 & 2,75 & 1,79 \\
\hline Baleares & 39 & 1,24 & 0,57 & 2,01 & 2,05 & 1,97 \\
\hline Canarias & 35 & 1,53 & 0,61 & 2,64 & 2,83 & 2,34 \\
\hline Cantabria & 30 & 1,28 & 0,58 & 1,87 & 2,67 & 1,48 \\
\hline Castilla y León & 46 & 1,36 & 0,35 & 2,23 & 2,09 & 2,41 \\
\hline Castilla-La Mancha & 51 & 1,25 & 0,41 & 2,21 & 2,12 & 2,33 \\
\hline Cataluña & 118 & 1,24 & 0,68 & 1,95 & 2,41 & 1,55 \\
\hline C Valenciana & 82 & 1,35 & 0,59 & 2,00 & 2,19 & 1,83 \\
\hline Extremadura & 29 & 1,30 & 0,54 & 2,04 & 1,97 & 2,15 \\
\hline Galicia & 58 & 1,20 & 0,54 & 2,03 & 2,42 & 1,69 \\
\hline Madrid & 136 & 1,19 & 0,54 & 1,89 & 2,23 & 1,63 \\
\hline Murcia & 38 & 1,33 & 0,29 & 2,84 & 3,43 & 2,47 \\
\hline Navarra & 94 & 1,37 & 0,72 & 1,85 & 1,95 & 1,82 \\
\hline País Vasco & 63 & 1,14 & 0,44 & 1,63 & 1,57 & 1,60 \\
\hline La Rioja & 35 & 1,18 & 0,66 & 1,69 & 1,84 & 1,50 \\
\hline Ceuta y Melilla & 30 & 1,49 & 0,48 & 2,55 & 2,75 & 2,30 \\
\hline Media (Total) & $(1086)$ & 1,29 & 0,53 & 2,10 & 2,32 & 1,92 \\
\hline
\end{tabular}

Fuente: INE. Encuesta de Empleo del Tiempo, 2009-10. Elaboración propia.

Tabla IV.

Primeros valores propios

\begin{tabular}{|lccc|}
\hline Número & Valor propio & Porcentaje & Porcentaje acumulado \\
\hline 1 & 3,8686 & 55,27 & 55,27 \\
2 & 1,0497 & 15,00 & 70,26 \\
\hline
\end{tabular}

Fuente: Elaboración propia.

Tabla V.

Correlación entre las variables y los primeros dos componentes principales

\begin{tabular}{|c|c|c|}
\hline Variables & Factor 1 & Factor 2 \\
\hline Ingresos del hogar & $-0,80$ & 0,07 \\
\hline Nivel de estudios (mujer) & $-0,91$ & 0,12 \\
\hline Número de hijos & 0,35 & 0,84 \\
\hline Nivel de estudios (hombre) & $-0,86$ & $-0,14$ \\
\hline Ratio (mujer/hombre) del tiempo dedicado a los hijos & 0,84 & 0,20 \\
\hline Ratio (mujer/hombre) del tiempo dedicado al hogar & 0,64 & $-0,49$ \\
\hline Ratio (mujer/hombre) del tiempo dedicado al trabajo remunerado & $-0,65$ & 0,16 \\
\hline Varianza explicada & $55,3 \%$ & $15,0 \%$ \\
\hline
\end{tabular}

Fuente: Elaboración propia.

El primer componente, que explica el $55,3 \%$ de la varianza, está formado por variables con coordenadas positivas y negativas, esto es, contrapone unos grupos de variables a otros. Concretamente, contrapone la ratio del tiempo dedicado al trabajo remunerado, el nivel de ingresos del hogar y el nivel de estudios a la ratio del tiempo dedicado al cuidado de los hijos y (en menor medida) del hogar. Esta contraposición sugiere una categorización de las regiones entre aquellas que tienen mejor situación socio-económica y una división del trabajo remunerado entre géneros más igualitaria y aquellas en las que la equidad de género en el reparto del trabajo doméstico es menor. El segundo componente explica una propor- 
ción bastante menor de la varianza (15\%) y opone las regiones con mayor número de hijos a las que tienen una mayor ratio del tiempo dedicado al trabajo en el hogar.

La interpretación de los componentes principales también puede realizarse mediante la representación gráfica de las cargas factoriales. La Figura I representa el círculo de correlación de las variables activas (con centro 0 y radio 1 ) sobre los primeros dos componentes (ejes). Los ejes representan el primer (horizontal) y segundo (vertical) componente principal. El ángulo entre dos variables representa la correlación entre ellas. La correlación es positiva si el ángulo es pequeño (las variables están próximas entre sí); no hay correlación lineal si el ángulo es de $90^{\circ}$, y la correlación es negativa si el ángulo es mayor de $90^{\circ}$. Cuanto más cerca se encuentra una variable del círculo de correlaciones, longitud de la flecha próxima a 1, mejor está recogida por los dos primeros componentes.

Figura I.

Círculo de correlación de las variables sobre el primer plano factorial

Factor 2: $15,0 \%$ varianza explicada

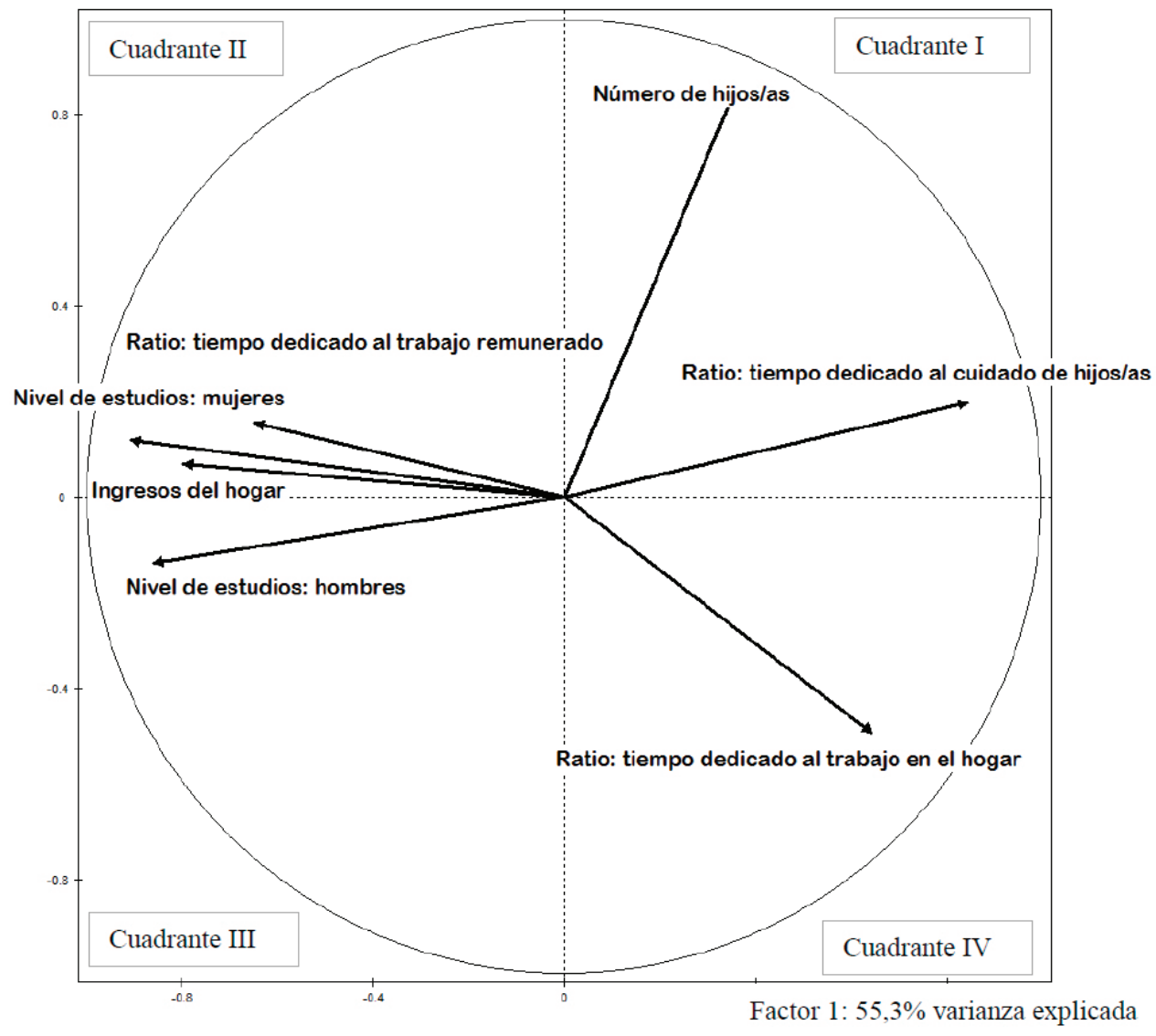

Fuente: Elaboración propia

La Figura II proyecta las regiones sobre los ejes formados por los dos primeros componentes. Con carácter general, las regiones que se encuentran en el primer cuadrante se caracterizan por tener familias con varios hijos, alta ratio de tiempo dedicado al cuidado de los hijos (y del hogar), bajas ratios de tiempo dedicado al trabajo remunerado y bajo nivel de estudios y de ingresos por hogar ${ }^{6}$.

En el segundo cuadrante destacan regiones con familias con varios hijos, alta ratio de tiempo dedicado al trabajo remunerado, alto nivel de ingresos y de educación y bajas ratios de tiempo dedicado al cuidado de los hijos. Son las regiones con la menor desigualdad de género.

Las regiones del tercer cuadrante tienen familias con pocos hijos, alta ratio de tiempo dedicado al trabajo remunerado, alto nivel de ingresos y de educación y baja ratio de tiempo dedicado al cuidado de los hijos.

Finalmente, en el cuadrante cuarto, encontramos regiones caracterizadas por tener familias con pocos hijos, baja ratio de tiempo dedicado al trabajo remunerado, bajo nivel de ingresos y de educación y elevadas ratios de tiempo dedicado al cuidado de los hijos y al hogar. 


\section{Resultados del análisis clúster}

A partir de la información obtenida en el ACP con los dos primeros factores extraídos ${ }^{7}$, se ha realizado un análisis clúster. Este análisis permite agrupar las regiones en cinco clases diferentes. Las regiones que pertenecen a una misma clase tienen características homogéneas respecto al reparto del tiempo entre hombres y mujeres y a otras características de los hogares e individuos que hemos considerado. Las regiones que pertenecen a clases diferentes presentan rasgos significativamente distintos. No obstante, es preciso señalar que las regiones dentro de una misma clase no comparten necesariamente todas las variables que caracterizan al grupo, aunque sí gran parte de ellas.

En la Figura A.Il del Apéndice se presenta el dendrograma que resulta de aplicar el método jerárquico aglomerativo de Ward. Se puede observar cómo partiendo del conjunto de regiones se distinguen dos grandes grupos, los cuales a su vez se van dividiendo en subgrupos. Hemos optado por extraer cinco grupos, al ser la opción que permitía obtener más matices relevantes desde la perspectiva de las desigualdades de género en el uso del tiempo. La Figura II proyecta sobre el primer plano factorial las regiones en los cinco grupos obtenidos. La Tabla VI recoge las variables activas e ilustrativas que caracterizan a cada grupo y la significación estadística de cada una de ellas.
El Clúster 1 está compuesto por cinco regiones: Cataluña, Madrid, Navarra, País Vasco y La Rioja. En estas regiones la brecha de género en relación con el trabajo doméstico y el cuidado de los hijos es menor que en el resto de los grupos. Las mujeres dedican al cuidado de sus descendientes un $61 \%$ más que sus parejas (en el conjunto de España esta diferencia es del 92\%). Si atendemos al tipo de tarea que realiza la pareja cuando están con sus hijos, observamos que este grupo de regiones presenta las menores diferencias de género en relación con las tareas de tipo rutinario. Las mujeres dedican a esta labor un $89 \%$ más que sus parejas, mientras que en el conjunto de España esta brecha es del 153\%. Respecto al trabajo en el hogar, las mujeres de este grupo dedican el doble de tiempo que sus parejas, elevándose este porcentaje en el conjunto del país al $132 \%$. Asimismo, este grupo presenta la menor brecha de género en el trabajo remunerado $(0,61)$. Además, el nivel educativo y los ingresos del hogar son más elevados que en la media del país.

Estas regiones registran las mayores tasas de actividad y empleo femeninas. Las mujeres reciben las mayores ganancias medias anuales (20.586 euros frente a los 18.642 euros en el conjunto de regiones) y el PIB per cápita y la renta bruta disponible (RBD) de los hogares supera a la del resto de grupos. Estas regiones también destacan por una fuerte participación de la industria en el valor añadido bruto (VAB).

Figura II.

Proyección de los individuos en el primer plano factorial (1-2). Clústeres

Factor 2: $15,0 \%$ varianza explicada

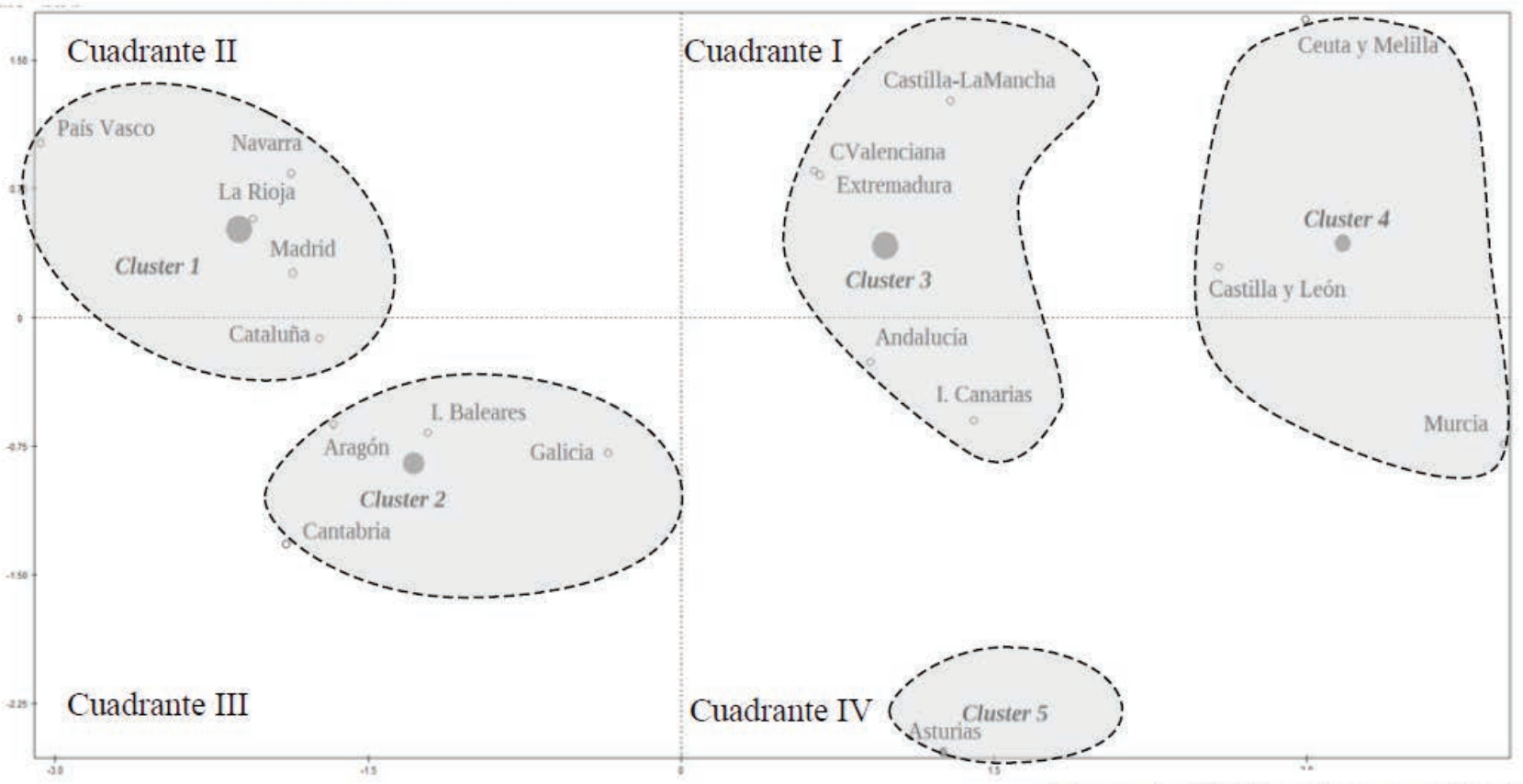

Factor 1: 55,3\% varianza explicada 
Desde la perspectiva de las políticas familiares y sociales, este grupo registra el mayor gasto per cápita en protección social y en servicios fundamentales (sanidad, educación y protección social). Las ayudas a los hogares y deducciones fiscales son también superiores al resto de los grupos.

El Clúster 2 incluye cuatro regiones: Aragón, Cantabria, Galicia e Islas Baleares. En este grupo, la brecha de género en el tiempo dedicado al trabajo renumerado y al cuidado de los niños, es algo menor que en el conjunto del Estado. No obstante, el tiempo dedicado a las tareas domésticas es similar a la media. La diferencia de género en las tareas rutinarias (tanto del hogar como de cuidado de los hijos) es menor que en la media de España. Los ingresos de los hogares en este grupo de regiones son superiores a la media.

Las magnitudes del mercado laboral que estamos considerando son algo más favorables que en el conjunto del país, y también es superior su nivel de riqueza (PIB per cápita y RBD). Respecto a las políticas familiares y sociales, las ayudas directas y deducciones fiscales son inferiores a las del clúster anterior, pero superiores al resto de los grupos.

El Clúster 3 está formado por cinco regiones: Andalucía, Extremadura, Castilla-La Mancha, Comunidad Valenciana e Islas Canarias. El patrón de distribución del tiempo que presenta este amplio grupo de regiones contribuye significativamente a definir el modelo que subyace en España. Es un patrón basado en la división tradicional de los roles de género, en el que el varón desempeña predominantemente el trabajo remunerado y la mujer muestra un alto sesgo hacía el trabajo doméstico. Esta pauta de comportamiento ha sido subrayada en otros trabajos (Sevilla-Sanz, Giménez-Nadal y Fernández 2010).

En este grupo los rasgos socio-económicos presentan algunas características propias. Las tasas de actividad y de empleo femenino son significativamente inferiores a la media española y a los grupos anteriores. Además, presentan una estructura productiva más orientada a la construcción, que aporta el 12,9\% del VAB (la media de España es del 11,4\%).

El gasto per cápita en servicios públicos fundamentales y en protección social es inferior a la media, pero superior al del Clúster 4. El mismo patrón siguen las ayudas familiares y las deducciones por hijo otorgadas por las administraciones regionales.

El Clúster 4 incluye tres regiones: Castilla-León, Murcia y Ceuta y Melilla. La característica más destacada de estas regiones es que las mujeres dedican más tiempo al cuidado de los hijos y del hogar que en los tres grupos anteriores. Las diferencias de género son particularmente elevadas en relación con las tareas rutinarias, tanto en el cuidado de los hijos como en las tareas del hogar, con unas ratios del 4,15 y 4,95 , respectivamente. La desigualdad de género también se deja notar en la distribución del tiempo dedicado al trabajo remunerado, dedicando las mujeres de este grupo un $63 \%$ menos de tiempo a esta actividad que los hombres. Asimismo, el nivel de estudios y los ingresos del hogar son inferiores al resto de los grupos.

La tasa de empleo y la ganancia media anual de las mujeres es la menor de todos los grupos. En cuanto a las políticas sociales, los recursos per cápita destinados a la protección social y a los servicios fundamentales son los más bajos de todos los grupos. Las ayudas y deducciones fiscales al hogar son, asimismo, inferiores a las del grupo anterior, pese a registrar la mayor tasa de fecundidad.

Las características propias de los hogares, el contexto socio-económico de estas regiones, así como el relativamente escaso apoyo institucional regional han favorecido el predominio del modelo tradicional de familia, que relega la mayor parte de la carga de trabajo doméstico a la mujer. Igual que ocurre en el clúster 3, el modelo patriarcal en el que el hombre es el principal breadwinner es el que mejor define a este grupo de regiones.

El Clúster 5 está formado exclusivamente por Asturias, que presenta un comportamiento outlier con características específicas. Registra valores extremos en tres de las variables activas: el número de hijos y el nivel de ingresos del hogar son significativamente bajos, mientras que la brecha de género en el trabajo remunerado muestra la mayor desigualdad de todos los grupos. En el resto de variables activas se encuentra en una posición intermedia entre los clústeres 3 y 4. De hecho, en el dendrograma se observa que comparte algunas características con estos clústeres.

Su mercado laboral se caracteriza por registrar la menor tasa de actividad y de empleo de todos los grupos, siendo particularmente baja entre las mujeres, lo que es compatible con la amplia desigualdad de género en el trabajo remunerado, anteriormente señalada. Por otro lado, los asturianos reciben las mayores prestaciones en servicios fundamentales y protección social. No obstante, estos conceptos están estrechamente relacionados con las jubilaciones anticipadas de los trabajadores de la minería (Pérez, Cucarela y Hernández 2015). Esto es consistente con una RBD de los hogares ligeramente superior a la media. Las ayudas a la conciliación y guarderías y las deducciones fiscales por hijos son, sin embargo, inferiores al resto de grupos.

\section{Conclusiones}

En este trabajo examinamos, desde una perspectiva regional, la relación entre los patrones de distribución del tiempo dedicado al trabajo remunerado y doméstico de las parejas heterosexuales y las características socioeconómicas e institucionales de las 
Tabla VI.

Resumen de resultados

\begin{tabular}{|c|c|c|c|c|c|c|}
\hline & Clúster1 & Clúster 2 & Clúster3 & Clúster4 & Clúster5 & Media \\
\hline \multicolumn{7}{|l|}{ Variables ilustrativas } \\
\hline \multicolumn{7}{|l|}{ Mercado laboral } \\
\hline Tasa de actividad de la mujer & $53,8^{\star *}$ & 51,4 & 48,9 & $47,6^{*}$ & $45,0^{* *}$ & 50,2 \\
\hline Ratio (mujer/hombre) tasa de actividad & $0,77^{* *}$ & $0,77^{\star *}$ & 0,72 & 0,70 & 0,76 & 0,74 \\
\hline Tasa de empleo de la mujer & $47,2^{* * *}$ & $44,5^{\star *}$ & $37,9^{* * *}$ & $38,0^{* * *}$ & $38,8^{* *}$ & 41,9 \\
\hline Ratio (mujer/hombre) tasa de empleo & $0,76^{\star *}$ & $0,76^{* *}$ & 0,69 & 0,66 & 0,74 & 0,72 \\
\hline Ganancia media anual en $€$ (mujeres) & $20.586^{\star *}$ & $18.121,8$ & $17.548^{\star \star *}$ & $17.458^{*}$ & $18.842,7$ & 18.642 \\
\hline Ratio (mujeres/hombres) ganancia media anual & $0,76^{*}$ & 0,78 & $0,81^{*}$ & 0,77 & 0,76 & 0,78 \\
\hline \multicolumn{7}{|l|}{ Economía } \\
\hline PIB per cápita (en €) & $28.804^{\star \star *}$ & 23.369 & $18.988^{* \star *}$ & $20.594^{\star \star}$ & 21.565 & 23.099 \\
\hline Renta Bruta Disponible de los hogares & $18.409^{* * *}$ & 15.733 & $12.704^{* * *}$ & 14.351 & 15.833 & 15.410 \\
\hline$\%$ de la agricultura en el VAB & 2,30 & 3,20 & 4,80 & 3,90 & 1,80 & 3,40 \\
\hline$\%$ de la industria en el VAB & $22,30^{* *}$ & 16,25 & $12,07^{* *}$ & 13,5 & 20,15 & 16,53 \\
\hline$\%$ de la construcción en el VAB & $10,14^{* * *}$ & 11,71 & $12,89^{*}$ & 9,98 & 13,47 & 11,41 \\
\hline$\%$ de los servicios en el VAB & 65,24 & 68,86 & 70,22 & 72,6 & 64,55 & 68,62 \\
\hline \multicolumn{7}{|l|}{ Políticas familiares y sociales } \\
\hline Gasto per cápita en servicios fundamentales (€) & 7.272 & 6.767 & $6.386^{\star * *}$ & $6.358^{*}$ & 8.255 & 6.816 \\
\hline Gasto per cápita en protección social $(€)$ & 4.333 & 4.009 & $3.572^{* \star *}$ & $3.485^{*}$ & 5.401 & 3.968 \\
\hline Ayudas familiares & $2,8^{* * *}$ & $2,5^{*}$ & $1,6^{*}$ & $1,3^{* *}$ & 2,0 & 2,1 \\
\hline Ayudas a la conciliación & 1,6 & 1,3 & 1,8 & 1,7 & 1,0 & 1,6 \\
\hline Ayudas guardería & 0,8 & 0,8 & 0,6 & 0,7 & 0,0 & 0,7 \\
\hline Deducción por hijos & $3,4^{* *}$ & 2,8 & $1,6^{* *}$ & $1,3^{* *}$ & 1,0 & 2,3 \\
\hline Tasa de fecundidad & $1,46^{*}$ & 1,30 & 1,36 & 1,66 & 1,06 & 1,41 \\
\hline \multicolumn{7}{|l|}{ Otras variables ilustrativas (ratio mujer/hombre) } \\
\hline $\begin{array}{l}\text { Tiempo dedicado al cuidado de los hijos (tareas no } \\
\text { rutinarias) }\end{array}$ & 1,15 & 1,04 & $1,32^{*}$ & ,83* & 89 & 1,11 \\
\hline $\begin{array}{l}\text { Tiempo dedicado al cuidado de los hijos (tareas } \\
\text { rutinarias) }\end{array}$ & $1,89^{* * *}$ & $2,22^{* *}$ & 2,52 & $4,15^{* * *}$ & 2,18 & 2,53 \\
\hline Tiempo dedicado a las tareas del hogar (no rutinarias) & ,91 & $1,26^{* *}$ & 1,02 &, $77^{* *}$ & ,93 & ,99 \\
\hline Tiempo dedicado a las tareas del hogar (rutinarias) & $2,70^{\star *}$ & $2,88^{*}$ & $3,01^{*}$ & $4,95^{*}$ & 3,86 & 3,26 \\
\hline \multicolumn{7}{|l|}{ Variables activas } \\
\hline Nivel de estudios (mujeres) & $2,64^{* * *}$ & $2,47^{*}$ & $2,25^{* *}$ & $2,09^{* * *}$ & 2,18 & 2,38 \\
\hline Nivel de estudios (hombres) & $2,42^{* *}$ & 2,31 & $2,13^{* * *}$ & $1,94^{* *}$ & 2,24 & 2,23 \\
\hline Nivel de ingresos del hogar & $3,13^{\star * *}$ & $3,20^{* *}$ & $2,56^{\star *}$ & $2,42^{* *}$ & 2,24 & 2,82 \\
\hline Número de hijos (variable codificada) & $1,53^{*}$ & $1,37^{\star * *}$ & $1,56^{*}$ & 1,64 & 1,27 & 1,51 \\
\hline $\begin{array}{l}\text { Ratio (mujer/hombre): tiempo dedicado al cuidado de } \\
\text { los hijos }\end{array}$ & $1,61^{* * *}$ & 1,76 & $2,09^{*}$ & $2,39^{\star \star *}$ & 1,79 & 1,92 \\
\hline $\begin{array}{l}\text { Ratio (mujer/hombre): tiempo dedicado a las tareas del } \\
\text { hogar }\end{array}$ & $2,00^{\star \star *}$ & 2,32 & 2,30 & $2,75^{*}$ & 2,75 & 2,32 \\
\hline $\begin{array}{l}\text { Ratio (mujer/hombre): tiempo dedicado al trabajo } \\
\text { remunerado }\end{array}$ &, $61^{*}$ &, $57^{* *}$ &, 54 &, $37^{* *}$ & ,35 &, 53 \\
\hline
\end{tabular}

Notas: Los datos de las columnas 1-5 son medias de las regiones que componen cada clúster. La última columna muestra la media para el conjunto de regiones. ${ }^{*},{ }^{* *},{ }^{* * *}$ denotan la significación estadística con respecto a la media de regiones. El Clúster 5 está compuesto únicamente por una región (Asturias), por lo que no disponemos de la significación estadística. Los valores relativos a las ayudas y deducciones fiscales de las CC. AA. se han obtenido como medias a partir de la Tabla II.

Fuente: Elaboración propia 
regiones españolas. Distinguimos, en el caso del trabajo doméstico, entre el tiempo dedicado a las tareas del hogar y al cuidado de los niños. Para ello, hemos combinado el Análisis de Componentes Principales (ACP) y el Análisis Clúster aplicados a los datos procedentes de la última oleada de la Encuesta de Empleo del Tiempo para el periodo 2009-2010.

El análisis identifica la existencia de cinco grupos de regiones con patrones diferentes de distribución del tiempo dedicado al trabajo. Estos patrones guardan relación con los contextos sociales, económicos e institucionales de las regiones, así como con las características educativas y económicas de los hogares. Este estudio pone de relieve que los grupos de regiones que presentan menores diferencias de género en la distribución del tiempo son aquellos con una mayor participación de la mujer en el mercado laboral, mayor riqueza económica y dotación de recursos destinados a un desarrollo más efectivo de las políticas de igualdad y sociales. Estos resultados son consistentes con la mayor parte de los estudios teóricos y empíricos y con nuestra hipótesis de partida.

En definitiva, el análisis realizado remarca la idea de que el contexto socioeconómico, laboral e institucional de las regiones está conectado con la distribución del tiempo entre géneros que subyace en los diferentes clústeres. Además, los resultados sugieren que el enfoque regional es un camino adecuado para estudiar la brecha de género en el uso del tiempo, al menos en países con una fuerte descentralización competencial, como es el caso de España.

Los resultados, por otro lado, también muestran similitudes entre las regiones que están en línea con las conclusiones obtenidas en los estudios por países, a saber, en todas las regiones las mujeres continúan siendo las principales responsables del trabajo doméstico y del cuidado de los hijos.
Además, en el caso de las mujeres que trabajan en el sector remunerado, la conciliación de la vida laboral y familiar sigue siendo una responsabilidad que recae mayoritariamente sobre ellas. En muchas ocasiones, las mujeres asumen un nuevo rol en el mundo laboral sin que se produzca una redistribución del trabajo doméstico, recayendo sobre ellas el mayor peso de estas tareas. Esta situación ha generado lo que se conoce como la segunda jornada laboral de muchas mujeres. Si a esto unimos la falta de flexibilidad en el mercado laboral (particularmente en el tiempo de trabajo) y la limitada provisión de servicios públicos para el cuidado de los hijos, la doble jornada supone para muchas mujeres una carga adicional a su trabajo extra-doméstico, además de una cortapisa para el desarrollo de su carrera profesional.

La evidencia empírica obtenida sugiere la necesidad de avanzar en el desarrollo de políticas nacionales y regionales dirigidas a favorecer la conciliación laboral y familiar, así como a mejorar la provisión de servicios públicos dirigidos al cuidado de los niños y personas mayores dependientes. Sería necesario consolidar un modelo de familia en la que no haya una separación entre los papeles de generador de ingresos familiares y cuidador, de forma que los hombres y las mujeres puedan asumir ambos roles en igualdad (Salazar 2016). El gobierno central y las administraciones regionales tienen un amplio recorrido para reducir las diferencias regionales de género que existen y alcanzar los estándares de los países europeos más avanzados a este respecto.

\section{Agradecimientos}

Los autores agradecen el apoyo financiero del Grupo de Investigación del Gobierno Vasco "Instituciones, Regulación y Política Económica" (IT1052-16).

\section{NOTAS}

1. Se utiliza el término región y Comunidad Autónoma indistintamente.

2. Se compara la distribución de hogares por regiones según nuestra sub-muestra y según los datos contenidos en el Censo elaborado por el INE. Se observa que no existen marcadas diferencias, salvo en los casos de Navarra, La Rioja y Ceuta y Melilla, donde existe una sobrerrepresentación. Consideramos que este hecho no altera los resultados, ya que son regiones pequeñas.

3. Para los datos relativos a estos factores se utiliza la media del periodo 2008-2010.

4. Lebart, Morineau y Piron (1995) y Escofier y Pagès (1990) detallan las particularidades de esta técnica estadística.

5. Según el Instituto de Política Familiar (2015) la tipología de las ayudas y deducciones regionales a las familias prácticamente no ha variado en los últimos años.
6. La representación de los individuos (regiones) difiere de la representación de las variables. Las variables están representadas por sus correlaciones mientras que los individuos están representados por sus proyecciones (Abdi y Williams 2010). Además, el análisis de individuos está centrado en el origen, mientras que, en el análisis de las variables, éstas están tipificadas.

7. La agregación realizada a partir del ACP considera una completa capacidad de compensación entre las variables, esto es, bajos valores en una variable pueden estar compensados por valores elevados en otra variable. Cuando esto ocurre, se puede inducir un sesgo no deseable en los resultados de la agregación (Ver Casadio y Guarini, 2013 para una detallada explicación sobre esta cuestión). 


\section{REFERENCIAS BibLIOGRÁficAs}

Aguiar, M. y E. Hurst. 2007. "Measuring Trends in Leisure: The allocation of Time over Five Decades." Quarterly Journal of Economics 122 (3): 969-1006. https://doi. org/10.1162/qjec.122.3.969

Abdi, H. y L.J. Williams. 2010. "Principal component analysis." Wiley Interdisciplinary Reviews: Computational Statistics 2 (4): 33-459. https://doi.org/10.1002/wics.101

Ajenjo, M. y J. García-Román. 2014. "Cambios en el uso del tiempo de las parejas. ¿Estamos en el camino de una mayor igualdad?." Revista Internacional de Sociología 72 (2): 453-476. https://doi.org/10.3989/ris.2012.05.28

Anxo, D., L. Mencarini, A. Pailhé, A. Solaz, L. Tanturri y L. Flood. 2011. "Gender Differences in Time Use over the Life Course in France, Italy, Sweden and US." Feminist Economics 17 (3): 159-195. https://doi.org/10.1080/13 545701.2011 .582822

Becker, G. 1981. A Treatise on the Family. Cambridge: Harvard University Press.

Bettio, F., M. Corsi, F.L. D'Ippoliti, A. Lyberaki, M.S. Lodovici y A. Vershchagina. 2013. The Impact of the Economic Crisis on the Situation of Women and Men and on Gender Equality Policies. Brussels: European Commission-Directorate General for Justice. https:// doi.org/10.2838/53123

Bittman, M., P. England, N. Folbre, L. Sayer y G. Matheson. 2003. "When does gender trump money? Bargaining and time in household work." American Journal of Sociology 109: 186-214. https://doi. org/10.1086/378341

Brines, J. 1994. "Economic Dependency, Gender, and the Division of Labor at Home". The American Journal of Sociology 100 (3): 652-688. https://doi. org/10.1086/230577

Burda, M, D.S. Hamermesh y P. Weil. 2013. "Total Work and Gender: Facts and Possible Explanations." Journal of Population Economics 26: 239-261. https://doi. org/10.1007/s00148-012-0408-x

Bustelo, M. 2014. "Three Decades of State Feminism and Gender Equality Policies in Multi-Governed Spain." Sex Roles 74: 107-120. https://doi.org/10.1007/ s11199-014-0381-9

Bustelo, M. y C. Ortbals. 2007. "The Evolution of Spanish State Feminism: A Fragmented Landscape". Pp. 201-223 en Changing State Feminism, editado por J. Outshoorn y J. Kantola. Basingstoke: Palgrave Macmillan. https:// doi.org/10.1057/9780230591424_11

Carrasco, C. y M. Dominguez. 2011. "Family Strategies for Meeting Care and Domestic Work Needs: Evidence from Spain." Feminist Economics 17 (4): 159-188. https://doi.org/10.1080/13545701.2011.614625

Casadio, E. y G. Guarini. 2013. "An unbalance adjustment method for development indicators." Social Indicators Research 112: 19-45. https://doi.org/10.1007/s11205012-0070-4

Coverman, S. 1985. "Explaining Husbands' Participation in Domestic Labor." Sociological Quarterly 26 (1): 81-97. https://doi.org/10.1111/j.1533-8525.1985.tb00217.x

Craig, L. 2006. "Parental Education, Time in Paid Work and Time with Children: An Australian Time-Diary Analysis". British Journal of Sociology 57 (4): 553-75. https://doi. org/10.1111/j.1468-4446.2006.00125.x

Craig, L. y M. Bittman. 2008. "The Incremental Time Costs of Children: An Analysis of Children's Impact on Adult
Time Use in Australia." Feminist Economics 14 (2): 59-88. https://doi.org/10.1080/13545700701880999

Davis, S. y T.N. Greenstein. 2004: "Cross-national variations in division of household labor". Journal of Marriage and Family 66: 1260-1271. https://doi.org/10.1111/j.00222445.2004.00091.x

Escofier, B. y J. Pagès. 1990. Analyses Factorielles Simples et Multiples. Paris: Dunod.

Esping-Andersen, G. 1990. The Three Worlds of Welfare Capitalism. London: Polity Press.

Esping-Andersen G., D. Boertien, J. Bonke y P. Gracia. 2013. "Couple specialization in Multiple Equilibria." European Sociological Review 29 (6): 1280-1294. https://doi. org/10.1093/esr/jct004

Esping-Andersen, G. 1999. Social foundations of postindustrial economies. Oxford: Oxford University Press.

Fundación BBVA-IVIE (Instituto Valenciano de Investigaciones Económicas). 2015. Gasto en los servicios públicos fundamentales en España y sus Comunidades Autónomas (2002-2013). Base de datos disponibles en internet: https://www.fbbva.es/bd/gasto-los-servicios-publicos-fundamentales-espana-comunidades-autonomas-2002-2013

Fuwa, M. 2004. "Macro-level gender inequality and the division of household labor in 22 countries." American Sociological Review 69: 751-767. https://doi. org/10.1177/000312240406900601

Gálvez-Muñoz, L., P. Rodríguez-Modroño, y M. DomínguezSerrano. 2011. "Work and Time Use By Gender: ANew Clustering of European Welfare Systems." Feminist Economics 17 (4): 125-157. https://doi.org/10.1080/1 3545701.2011 .620975

Geist, C. 2009. "Two Worlds of Housework? Examining Employed Single and Partnered Women in the Decade after Unification." Journal of Comparative Family Studies 40 (3): 415-437.

Gimenez-Nadal, J.I. y A. Sevilla. 2012. "Trends in Time Allocation: A Cross-Country Analysis." European Economic Review 56: 1338-1359. https://doi. org/10.1016/j.euroecorev.2012.02.011

Gimenez-Nadal, J.I. y A. Sevilla. 2014. "Total Work Time in Spain: Evidence from Time Diary Data." Applied Economics 46 (16): 1894-1909. https://doi.org/10.108 0/00036846.2014.887194

Gracia, P. y G. Esping-Andersen. 2015. "Fathers' child care time and mothers' paid work: Across-national study of Denmark, Spain, and the United Kingdom". Family Science 6 (1): 270-281. https://doi.org/10.1080/19424620.2015.1082336

Greenstein, T. N. 1996. "Husbands' participation in domestic labor: Interactive effects of wives' and husbands' gender ideologies." Journal of Marriage and the Family 58: 585-595. https://doi.org/10.2307/353719

Gutierrez-Domenech, M. 2005. "Employment after motherhood: a European comparison". Labour Economics 12 (1): 99123. https://dx.doi.org/10.1016/j.labeco.2004.04.004

Hook, J.L. 2006. "Care in context: men's unpaid work in 20 countries, 1965-2003". American Sociological Review 71: 639-660. https://dx.doi. org/10.1177/000312240607100406

Hook, J.L. 2010. "Gender inequality in the welfare state: sex segregation in housework, 1965-2003." American Journal of Sociology 115 (5): 1480-1523. https://dx.doi. org/10.1086/651384 
Instituto de Política Familiar. 2015. Las ayudas fiscales a las familias en las Comunidades Autónomas. Instituto de Política Familiar.

Killewald, A. y M. Gough. 2010. "Money isn't everything: Wives' earnings and housework time." Social Science Research 39: 987-1003. https://dx.doi.org/10.1016/j. ssresearch.2010.08.005

Lahey, K.A. y P. De Villota. 2013. "Economic crisis, gender equality, and policy responses in Spain and Canada." Feminist Economics 19 (3): 82-107. https://doi.org/10. $1080 / 13545701.2013 .812267$

Lebart, L., A. Morineau y M. Piron. 1995. Statistique Exploratoire Multidimensionnelle. Paris: Dunod.

Lewis, J. 2009. Work-family balance, gender and policy. Cheltenham: Edward Elgar.

Lundberg, S. y R.A. Pollac. 1996. "Bargaining and distribution in marriage." Journal of Economic Perspectives 10: 139-158. https://doi.org/10.1257/jep.10.4.139

Manser, M, y M. Brown. 1980. "Marriage and Household DecisionMaking: A Bargaining Analysis." International Economic Review 21 (1): 31-44. https://doi.org/10.2307/2526238

McElroy, M.B. y M.J. Horney. 1981. "Nash-Bargained Household Decisions: Toward a Generalization of the Theory of Demand." International Economics Review 22 (2): 333-349. https://doi.org/10.2307/2526280

Orloff, A. 1996. "Gender in the Welfare State." Annual Review of Sociology 22: 51-78. https://doi.org/10.1146/annurev.soc.22.1.51

Perez García, P., T. Cucarela Vicent y L. Hernández Lahiguera. 2015. "Servicios públicos, diferencias territoriales e igualdad de oportunidades." Fundación BBVA-Ivie (Instituto Valenciano de Investigaciones Económicas). Disponible en: https://www.fbbva.es/wp-content/ uploads/2017/05/dat/INFORME SERVICIOS PUBLICOS_FUNDAMENTALES\%20_FBBVA-IVIE.pd

Phipps, S., P. Burton y L. Osberg. 2011. "Time as a source of inequality within marriage: are husbands more satisfied with time for themselves than wives?" Feminist economics 7 (2): 1-21. https://doi. org/10.1080/1354700110068261

Ruppanner, L. y D.J. Maume. 2016. "The state of domestic affairs: Housework, gender and state-level institutional logics." Social Science Research 60: 15-28. https://doi. org/10.1016/j.ssresearch.2016.04.006

Sainsbury, D. 1996 Gender, Equality and Welfare States. Cambridge University Press.

Salazar Benítez, O. 2016. "The Fragility of Gender Equality Policies in Spain." Social Sciences 5 (2): 1-17. https:// doi.org/10.3390/socsci5020017

Sevilla-Sanz, A., J. I. Giménez-Nadal y C. Fernández. 2010. "Gender roles and the division of unpaid work in Spanish Households." Feminist Economics 16 (4): 137184. https://doi.org/10.1080/13545701.2010.531197

Stevens J. 1986. Applied Multivariate Statistics for the Social Sciences. Hillsdale, NJ: Lawrence Erlbaum Associates.

West, C. y D. Zimmerman. 1987. "Doing gender." Gender \& Society 1 (2): 125-151. https://doi. org/10.1177/0891243287001002002

\section{APÉNDICE I}

Figura A.I.

Dendograma

Hierarchical Cluster Analysis (on factors)

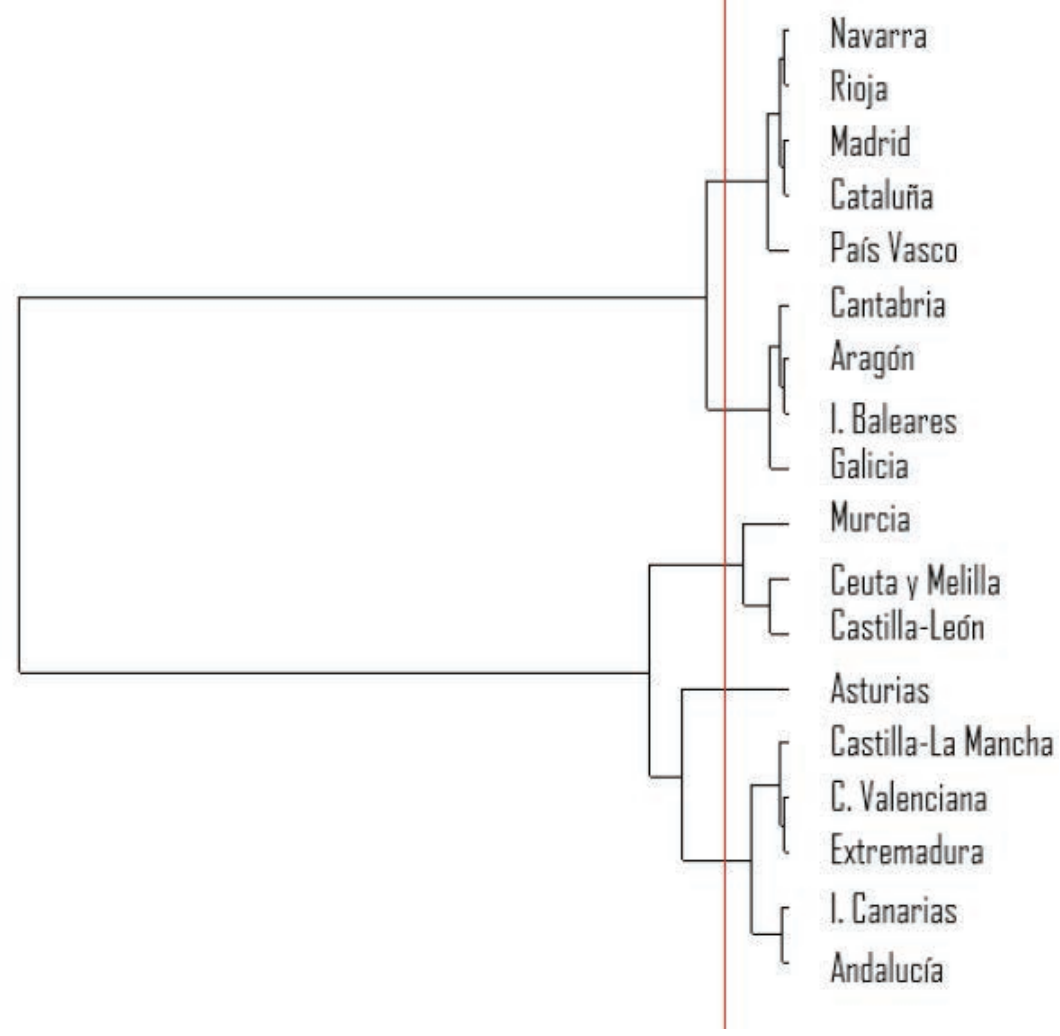


Tabla A.I. Variables ilustrativas

\begin{tabular}{|c|c|c|}
\hline Variables ilustrativas & Media & D.S \\
\hline \multicolumn{3}{|l|}{ Mercado laboral (2008-2010) } \\
\hline Tasa de actividad & 59,07 & 3,79 \\
\hline Ratio (mujer/hombre) tasa de actividad & 0,74 & 0,04 \\
\hline Tasa de empleo & 50,00 & 4,04 \\
\hline Ratio (mujer/hombre) tasa de empleo & 0,72 & 0.06 \\
\hline Tasa paro & 15,34 & 4,39 \\
\hline Ganancia media anual mujeres (en $€$ ) & 18642,3 & 1579,2 \\
\hline Ratio (mujeres/hombres) ganancia media anual & 0,78 & 0,04 \\
\hline \multicolumn{3}{|l|}{ Economía (2008-2010) } \\
\hline PIB per cápita & 23099,0 & 3526,1 \\
\hline Renta disponible bruta de los hogares (per cápita) en $€$ & 15410,6 & 2415,7 \\
\hline Participación de la Agricultura en el VAB & 3,45 & 2,52 \\
\hline Participación de Industria en el VAB & 16,53 & 6,39 \\
\hline Participación de Construcción en el VAB & 11,41 & 1,83 \\
\hline Participación de Servicios en el VAB & 68,62 & 7,84 \\
\hline \multicolumn{3}{|l|}{ Políticas familiares y sociales (2008-2010) } \\
\hline Gasto per cápita en servicios públicos fundamentales (educación, sanidad y protección social) (€) & 6816,7 & 749,9 \\
\hline Gasto per cápita en protección social $(€)$ & 3968,3 & 636,4 \\
\hline Ayudas familiares & 2,1 & ,83 \\
\hline Ayudas a la conciliación & 1,5 & ,61 \\
\hline Ayudas guardería & ,7 & ,48 \\
\hline Deducción por hijos & 2,3 & 1,2 \\
\hline Tasa de fecundidad. $N^{\circ}$ medio de hijos por mujer en edad fértil (15-49 años) & 1,4 & 0,25 \\
\hline \multicolumn{3}{|l|}{ Otras variables ilustrativas: (Ratio mujer/hombre) } \\
\hline Tiempo dedicado al cuidado de los hijos (tareas no rutinarias) & 2,53 & 0,81 \\
\hline Tiempo dedicado al cuidado de los hijos (tareas rutinarias) & 1,11 & 0,28 \\
\hline Tiempo dedicado a las tareas del hogar (tareas no rutinarias) & 3,27 & 1,03 \\
\hline Tiempo dedicado a las tareas del hogar (tareas rutinarias) & 1,00 & 0,25 \\
\hline
\end{tabular}

Fuente: Elaboración propia

\section{APÉNDICE II}

Para evaluar la significatividad del modelo factorial existen varios contrastes, entre los que destacan la prueba de esfericidad de Bartlett y el índice de Kaiser-Meyer-Olkin (Tabla A.II). La prueba de Bartlett evalúa la correlación entre las variables objeto de estudio. El valor obtenido (83,028; Sig. 0,000) muestra que podemos rechazar la hipótesis de no correlación entre las variables y afirmar que hay, al menos, un factor común que motiva la aplicación del análisis factorial. El índice de Kaiser-Meyer-Olkin (KMO) es una medida de adecuación muestral de las variables. El valor de esta medida $(0,6676)$ excede sensiblemente el valor mínimo sugerido para aplicar el análisis de componentes principales.

Tabla A.II. KMO y Prueba de Barlett

\begin{tabular}{|lll|}
\hline Medida de adecuación muestral de Kaiser-Meyer-Olkin & & 0,6676 \\
\hline Prueba de esfericidad de Bartlett & Approx. Chi-Square (25) & 69,66 \\
& Sig. & 0,0000 \\
\hline
\end{tabular}

El índice de KMO varía entre 0 y 1 . Se consideran adecuados los valores superiores a 0,5.

Fuente: Elaboración propia 
AMAIA ALTUZARRA ARTOLA es Profesora Titular de Universidad en la Universidad del País Vasco, España. Su investigación se centra en el estudio de la competitividad y del mercado de trabajo con especial atención a las diferencias de género. Algunos artículos están publicados en Industrial and Labor Relations Review, Open Economics, Journal of Housing, Transition Studies, Acta Oeconomica o Journal of Post Keynesian Economics. También es autora de diversos libros y capítulos de libros.

CATALINA GÁLVEZ GÁLVEZ es Doctora y Profesora Titular de Universidad en la Universidad del País Vasco, España. Su investigación se centra en el área del mercado de trabajo, el desarrollo urbano y regional y el de las políticas macroeconómicas. Ha publicado artículos en revistas como Panoeconomicus, Tourism Economics o Scripta Nova. También ha publicado diversos libros y capítulos de libros.

ANA GONZÁLEZ FLORES es Doctora y Profesora Titular de Universidad en la Universidad del País Vasco, España. Sus investigaciones están centradas en el área de las políticas macroeconómicas, en el desarrollo urbano y regional y en el mercado de trabajo. Ha publicado artículos en revistas como Panoeconomicus, Tourism Economics, Municipal Engineer o African Journal of Business Managment. También ha publicado diversos libros y capítulos de libros. 